\title{
A case of haemophagocytic lymphohistiocytosis (HLH)
}

\author{
T M R Gunasekera ${ }^{1}$, Srilal de Silva ${ }^{2}$, H M S Vidyatilaka ${ }^{3}$, M A M Faizal ${ }^{4}$
}

Sri Lanka Journal of Child Health, 2008; 37: 98-99

(Key words: Haemophagocytic lymphohistiocytosis, HLH)

\section{Introduction}

Haemophagocytic lymphohistiocytosis (HLH) results from infiltration of various organs and tissues with lymphocytes, natural killer (NK) cells and haemophagocytic histiocytes and reflects a highly stimulated yet ineffective immune response triggered by various stimuli ${ }^{1}$. It is classified as Class 11 Histiocytosis according to Histiocyte Society Classification (Writing Group of the Histiocyte Society 1987)².

A high degree of clinical suspicion is needed in the initial diagnosis of this condition as clinical features and laboratory investigations are non specific. If treatment is not established immediately, death supervenes due to severe sepsis and multi organ dysfunction.

\section{Case report}

A previously healthy 11 year old boy presented to hospital with fever of 14 days duration associated with respiratory symptoms at the onset. The illness progressed rapidly with jaundice and tea coloured urine appearing on day 5 and a generalized purpuric rash on day 8 . Towards the end of the second week, generalized oedema was noticed with deepening of jaundice. He was admitted to the intensive care unit of the Lady Ridgeway Hospital with diffuse mucocutaneous bleeding, deterioration of intellect and circulatory collapse. $\mathrm{He}$ is the second born of healthy non consanguineous parents and the family history was insignificant.

Examination revealed a well built boy with severe pallor, deep icterus, generalized oedema, multiple mucosal and skin bleeds. The abdominal examination revealed gross

${ }^{1}$ Senior Registrar in Paediatrics, ${ }^{2}$ Consultant Paediatrician, Paediatric \& Neonatal Intensive Care Unit, ${ }^{3}$ Consultant Haematologist, ${ }^{4}$ Senior Registrar in Paediatric Intensive Care, Lady Ridgeway Hospital, Colombo.

(Received on 28 May 2007. Accepted on 20 June 2007) hepatosplenomegaly with free fluid. His Glasgow coma scale ranged between 8 and 10 . Investigations revealed a haemoglobin of $4 \mathrm{~g} / \mathrm{dl}$ and a white blood cell count of $4.2 \times 10^{9} / \mathrm{L}$ of which $18 \%$ were neutrophils and $78 \%$ lymphocytes. Platelet count was $5 \times 10^{9} / \mathrm{L}$. Reticulocyte count was $0.4 \%$. D dimers were $1.2 \mathrm{mg} / \mathrm{dl}$ (normal $<0.2$ ). Urine and blood cultures were sterile. Serology did not identify any viral titres. Mycoplasma antibodies were negative. His liver functions were severely disturbed with an INR $>6$ and serum albumin of $17 \mathrm{~g} / \mathrm{l}$. Renal function tests revealed hyponatraemia with elevated serum creatinine. The bone marrow aspiration was aparticulate with severely depressed erythroid and granulocytic precursors and absent megakaryocytes. There were no blasts/infiltrates. The macrophages were moderately increased with mild dysplasia and gross haemophagocytosis compatible with reactive HLH.

$\mathrm{He}$ was treated with intravenous cefotaxime, amikacin, penicillin and liver failure regime as well as IPPV ventilation. He received IV immunoglobulins, recombinant activated factor V11 injections and several transfusions of platelets, plasma and blood. Despite treatment his condition deteriorated further and he succumbed on day 18 of the illness.

\section{Discussion}

HLH occurs in both familial and acquired forms and both can be triggered ${ }^{1}$. The primary familial form has autosomal recessive inheritance and a frequency of 1 per 50000 births. $70 \%$ of affected patients manifest symptoms during the first year. There is a preceding symptom free period and HLH is the only clinical manifestation. The secondary familial variety occurs in association with immune deficiency syndromes such as Chediak Higashi syndrome, Griscelli syndrome and $\mathrm{X}$ linked lymphoproliferative disease ${ }^{1}$.

The acquired forms are mainly due to infections, most commonly viruses of the herpes group. Epstein Barr virus (EBV) has been isolated in 
$74 \%$ of those with a viral aetiology $y^{3}$. Bacteria, fungi and protozoa, especially leishmania are non-viral infective causes. The macrophage activating syndrome (MAS) is a form of acquired HLH occurring in association with autoimmune diseases like rheumatoid arthritis and SLE, and is triggered by infections as well as drugs such as NSAIDs and methotrexate. It occurs during the active phase of the disease and carries a fatality of $10-20 \%$. HLH is also being reported with malignancies, especially lymphomas and metabolic diseases like lysinuric protein intolerance and multiple sulfatase deficiency.

In the pathogenesis of HLH, the infiltrated cytotoxic $\mathrm{T}$ cells and NK cells hyper secrete proinflammatory cytokines like interferon gamma, tumor necrosis factor alfa, interleukin6, 10 and macrophage colony stimulating factor which lead to tissue necrosis and organ failure. Despite the accumulation of these cells at tissues, their cytotoxic activity is impaired due to defective cytolytic granules containing perforin and granzyme as well as improper immunological synapsing ${ }^{4}$.

The 3 cardinal features of HLH are high grade fever, hepatosplenomegaly and pancytopenia. Lymphadenopathy, hepatic impairment with coagulopathy and severe sepsis are additional features. CNS involvement occurs in up to $73 \%^{5}$ and in $20 \%$ is present at the onset ${ }^{6}$. The neurological features include hemiplegia, cranial nerve palsies, focal deficits and seizures, especially myoclonic ${ }^{7}$.

Characteristic laboratory findings include elevated serum triglycerides, ferritin, and decreased fibrinogen levels. Bone marrow examination reveals infiltration of morphologically benign histiocytes with erythrophagocytosis, which may be absent initially. The cerebrospinal fluid of affected patients shows increased proteins, lymphocytes, foamy histiocytes and occasional erythrophagocytes. Neuroimaging findings are nonspecific. The brain biopsy shows characteristic infiltration of haemophagocytic histiocytes at autopsy. The immunological assay of impaired NK cell activity is diagnostic. Screening for triggers is vital as some conditions are treatable.

Management is mainly directed towards suppression of hyperinflammation and various regimes have been tried. IV immunoglobulins and IV dexamethazone are used initially. Cyclosporin A, etoposide and antithymocytic globulin are second line drugs. CNS disease warrants intrathecal methotrexate. Stem cell transplantation is beneficial in familial cases ${ }^{4}$. Treatment of triggers as well as vigorous supportive management improves outcome. Despite treatment, the prognosis of both familial and acquired forms of HLH is usually poor and is rapidly fatal in untreated cases. The CNS disease can cause relapses and irreversible disability.

\section{References:}

1. Familial and acquired hemophagocytic lymphohistiocytosis European Journal of Pediatrics, 2007; 166 (2): 95-109

2. Writing Group of the Histiocyte Society. Histiocytosis syndromes in children. Lancet. 1987; 1(8526):208-9.

3. Janka G, Imashuku S, Elinder G, et al. Infection and malignancy associated hemophagocytic syndromes. Secondary hemophagocytic lymphohistiocytosis. Hematol Oncol Clin North Am 1998; 12: 435-44.

4. Janka GE, Schneider EM. Modern management of children with hemophagocytic lymphohistiocytosis. $\mathrm{Br} \mathrm{J}$ Haematol 2004; 124:4-14.

5. Haddad E, Sulis ML, Jabado N, et al. Frequency and severity of central nervous system lesions in hemophagocytic lymphohistiocytosis. Blood 1997; 89:794800.

6. Henter JI, Elinder G. Cerebromeningeal hemophagocytic lymphohistiocytosis. Lancet 1992; 339:104-7.

7. Kollias SS, Ball WS, Tzika AA, Harris RE. Familial lymphohistiocytosis: neuroradiologic evaluation with pathologic correlation. Radiology 1994; 192:743-54. 